\title{
PEP-CMV Vaccine
}

National Cancer Institute

\section{Source}

National Cancer Institute. PEP-CMV Vaccine. NCI Thesaurus. Code C107243.

A peptide vaccine derived from cytomegalovirus (CMV) antigens with potential immunostimulating activity. Intradermal administration of the PEP-CMV vaccine may stimulate the immune system to mount a specific helper and cytotoxic T-lymphocyte (CTL) response ag ainst CMV-infected tumor cells. Infection with the herpesvirus CMV may play a significant role in tumor cell initiation and progression as well as chemoresistance. 